\title{
Asociación entre meningitis y punción dural en ratas bacterémicas. Un análisis crítico
}

\author{
The Association between meningitis and dural puncture in \\ bacteremic rats. A critical analysis
}

Lorena Basso V., MD. ${ }^{1}$, Héctor J. Lacassie, MD, ${ }^{2}$

\begin{abstract}
Introduction: The presence of an active bacteremia has been considered a relative contraindication to perform an intrathecal puncture due to the risk of causing meningitis or epidural abscess. However, a clear and definite causal relationship has not yet been demonstrated. Objectives: To determine the relationship between intrathecal puncture and the development of meningitis in subjects with bacteremia. Materials and Methods: Experimental study in rats with chronic bacteremia to which dural puncture was performed. Meningitis was then evaluated by direct drainage of the cisterna magna and histopathological studies of brain tissue. Results and Conclusion: 12 of 40 bacteremic rats that underwent intrathecal puncture developed meningitis. Previous administration of antibiotics seems to reduce this risk.
\end{abstract}

\section{RESUMEN}

Introducción: Se ha considerado la presencia de una bacteremia activa como contraindicación relativa para realizar una punción intratecal por el riesgo de provocar meningitis o absceso peridural. Sin embargo, aún no se ha podido demostrar una relación causal clara y definida. Objetivos: Determinar la relación entre punción intratecal y el desarrollo de meningitis en sujetos con bacteremia. Materiales y Métodos: Estudio experimental en ratas con bacteremia crónica a las que se les realizó punción dural, luego se evaluó la presencia o no de meningitis mediante drenaje directo de la cisterna magna y estudios

\section{Key words:}

Meningitis,

bacterial meningitis,

bacteremia,

fever,

spinal anesthesia,

spinal puncture,

central nervous system

infections

\section{Palabras clave:}

Meningitis, meningitis bacterianas, bacteremia, infección, fiebre, anestesia raquídea, punción espinal

Residente de Anestesiología, Unidad de Anestesiología, Escuela de Medicina, Facultad de Medicina, Universidad de La Frontera.

2 Profesor Asociado en Anestesiología, División de Anestesiología, Escuela de Medicina, Facultad de Medicina, Pontificia Universidad Católica de Chile.

Fecha de ingreso: 10 de abril de 2019

Fecha de aceptación: 12 de abril de 2019

ORCID

https://orcid.org/0000-0001-5758-4113

Correspondencia:

Hector J. Lacassie

lacassie@med.puc.cl 
histopatológicos de tejido cerebral. Resultados y Conclusión: 12 de 40 ratas bacterémicas a las que se le realizó punción intratecal desarrollaron meningitis. La administración previa de antibiótico parece disminuir este riesgo.

\section{Introducción}

te trabajo presenta un análisis crítico del artículo de Carp y cols[1],[2].

La anestesia neuroaxial, tanto peridural como espinal, es actualmente la forma más común de anestesia regional[3]. Esta última es además, la técnica de elección para operación cesárea en muchos centros del mundo[4]. A lo largo del tiempo se ha considerado la presencia de infección sistémica como una contraindicación relativa para este procedimiento, ya que al introducir la aguja al espacio peridural o subaracnoideo, ésta podría arrastrar sangre infectada con el consecuente desarrollo de meningitis o absceso peridural. Sin embargo, hasta ahora no se ha podido demostrar una relación causal definitiva entre estos dos factores. De hecho, realizar una punción lumbar diagnóstica sigue siendo parte de la práctica clínica habitual en el enfrentamiento de pacientes febriles o con bacteremia de origen desconocido[5]. La evidencia actual es contradictoria y en su mayoría proviene de reportes de casos e investigaciones basadas en animales, cuya metodología difiere entre sí.

Pregunta clínica: En pacientes cursando una bacteremia documentada, ¿existe asociación entre punción intratecal y el desarrollo de meningitis?

\section{Métodos}

Unidad experimental e intervención: se emplearon ciento cuarenta ratas de la cepa Sprague-Dawly macho. Se aplicó un modelo probado de bacteremia crónica[6]. Luego se distribuyeron en 4 grupos: Grupo A: cuarenta ratas inoculadas con $10^{9}$ unidades formadoras de colonias (UFC) de Escherichia coli ( $E$. coli), con bacteremia confirmada mediante hemocultivo. Se anestesiaron y se sometieron a una punción percutánea de la cisterna magna, bajo técnica estéril con una aguja espinal 26 G. 24 horas después de la punción inicial se les extrajo quirúrgicamente líquido cefalorraquideo (LCR) mediante exposición y drenaje directo y se cultivó bajo condiciones estándar. Luego del drenaje, se extrajo cerebro y médula espinal para análisis histológico en busca de infección. Grupo B: cuarenta animales bacterémicos que no se les reali- zó punción intratecal. Grupo C: treinta animales sin bacteremia y puncionados intratecalmente. Grupo $D$ : treinta animales bacterémicos que recibieron tratamiento antibiótico 15 minutos previo a la punción intratecal.

El desenlace primario fue el desarrollo de meningitis en animales bacterémicos posterior a la punción intratecal. El secundario: desarrollo de meningitis en animales bacterémicos que no fueron sometidos a punción intratecal (grupo B) y en sujetos que recibieron una dosis de antibiótico previo a la punción intratecal percutánea (grupo D).

\section{Resultados}

Desenlace primario: 12 de 40 ratas bacterémicas a las que se le realizó punción intratecal (Grupo A) desarrollaron meningitis. Desenlaces secundarios: tanto las ratas bacterémicas que no fueron puncionadas (grupo B) como las sin bacteremia, pero puncionadas intratecalmente (Grupo C, control) no desarrollaron meningitis. Las 30 ratas bacterémicas a las que se les administró antibiótico 15 minutos antes de la punción intratecal (grupo D), no desarrollaron meningitis.

\section{Conclusión}

Los resultados muestran que la punción intratecal se asocia a desarrollo de meningitis en ratas bacterémicas al momento de la punción. Sin embargo, la administración de antibiótico previo, parece atenuar este riesgo.

\section{Análisis crítico}

Validez interna: hay una descripción breve de criterios de inclusión. No se mencionan los criterios de exclusión. No hubo cálculo del tamaño muestral, aleatorización de las unidades de estudio o ciego. Esta falta de información puede ser fuente de sesgo, aunque en estudios experimentales en laboratorio, estos factores habitualmente están controlados. Si bien el seguimiento fue completo de los sujetos en estudio, 
no se realizó análisis por intención a tratar y sólo se incluyó en el análisis al total de animales que completaron el estudio. El criterio para el diagnóstico final de meningitis está bien definido y se explica adecuadamente. Todos los análisis fueron hechos en un centro especializado y certificado.

Validez externa: el tipo de sujetos incluidos en el estudio corresponde a ratas utilizadas en estudios de experimentación, lo que estandariza el modelo pero dificulta su generalización en humanos. Otras consideraciones a tener en cuenta son: el agente utilizado como modelo de bacteremia ( $E$. coli) es un agente causal poco frecuente de meningitis; el sitio de punción percutánea escogido difiere del utilizado clínicamente en anestesia y el tamaño del trócar utilizado en la punción generaría un traumatismo proporcionalmente mayor que en humanos. Además, la punción intratecal es un procedimiento que por lo general incluye el uso de anestésicos locales, a los cuales se les ha atribuido una capacidad bacteriostática significativa, no utilizados en este estudio. Finalmente, los autores conocían el antibiograma de la cepa infecciosa, por lo que su tratamiento antibiótico era conocidamente efectivo, lo que habitualmente no ocurre en la clínica.

\section{Comentario}

Este estudio fue realizado hace un cuarto de siglo y aún mantiene actualidad dada la situación clínica que plantea y que nos podemos ver enfrentados a diario. Si bien hay aspectos de difícil traducción al contexto clínico, es una buena guía y ejercicio intelectual.

Es un estudio con adecuada validez interna, con seguimiento completo de los sujetos y criterios claros y bien definidos. Si bien hay aspectos que no se mencionan y que podrían dar lugar a sesgo, en este tipo de diseños todas estas variables están por lo general controladas. Es un estudio con limitada validez externa, dada principalmente por las diferencias que existen en cuanto al modelo estudiado (animal) y al que se desea extrapolar (humanos), lo que hace difícil su interpretación. Además, los sujetos presentaban signos clínicos de sepsis temprana, lo que difiere clínicamente con aquellos pacientes que pueden presentar bacteremia de bajo grado (particularmente obstétricas y urológicos).

El estudio provee información valiosa sobre el probable beneficio de iniciar tratamiento antibiótico en pacientes febriles previo a una anestesia espinal, si el beneficio de la técnica lo amerita, como es el caso de las pacientes obstétricas. Habría sido interesante agregar un quinto grupo donde se administraran antibióticos posterior a la punción intratecal[2], en especial para los casos en que se realiza una punción lumbar diagnóstica en que no se puede administrar antibióticos previo al cultivo de LCR.

\section{Referencias}

1. Carp H, Bailey S. The association between meningitis and dural puncture in bacteremic rats. Anesthesiology. 1992 May;76(5):739-42. https:// doi.org/10.1097/00000542199205000-00012 PMID:1575342

2. Chestnut DH. Spinal anesthesia in the febrile patient. Anesthesiology. 1992 May;76(5):667-9. https:// doi.org/10.1097/00000542199205000-00001
PMID:1575331

3. Zorrilla-Vaca A, Healy RJ, RiveraLara L, Grant MC, Maragakis LL, Escandón-Vargas K, et al. Epidemiology of septic meningitis associated with neuraxial anesthesia: a historical review and meta-analysis. Minerva Anestesiol. 2018 Mar;84(3):36377. PMID:29108403

4. Lyons G, Akerman N. Problems with general anaesthesia for Caesarean section. Minerva Anestesiol. 2005 Jan-Feb;71(1-2):27-38. PMID:15711504
5. Wedel DJ, Horlocker TT. Regional anesthesia in the febrile or infected patient. Reg Anesth Pain Med. 2006 Jul-Aug;31(4):324-33. https:// doi.org/10.1097/00115550200607000-00007 PMID:16857552

6. Mela-Riker L, Alexander $P$, Bartos D, Bryant RE, Connell RS, Erwin L, et al. Chronic hyperdynamic sepsis in the rat: I. Characterization of the animal model. Circ Shock. 1988 Aug;25(4):231-44. PMID:3048769 